\title{
Conversion of data from the laser scanner to the Ansys Workbench
}

\author{
Ondrej Štalmach ${ }^{1, *}$, Alžbeta Sapietová ${ }^{1}$, Vladimír Dekýš ${ }^{1}$, Peter Šulka ${ }^{1}$, Lukáš Gajdoš ${ }^{2}$ \\ ${ }^{1}$ Department of Applied Mechanics, Faculty of mechanical engineering, University of Žilina, \\ Univerzitná 8215/1, 01026 Žilina, Slovak Republic \\ ${ }^{2}$ Schaffler Slovakia, Dr G Schaefflera 1, Kysucke Nove Mesto, Slovak Republic
}

\begin{abstract}
This paper deals with the data processing in the form of a cloud of points scanned using a handheld $3 \mathrm{D}$ laser scanner. The aim of this paper is to use this data to create a representative FEM model and compare two different approaches while one of them involves creating a CAD model and the other one not. The data processing is carried out in the freeware systems MeshLab and Meshmixer and FEM analysis in the software ANSYS Workbench.
\end{abstract}

Keywords: 3D laser scanner, data processing, CAD model, MeshLab, Ansys Workbench

\section{Introduction}

Reverse engineering is very common in such diverse fields as software engineering, entertainment, automotive, consumer products, microchips, chemicals, electronics, and mechanical designs. For example, when a new machine comes to market, competing manufacturers may buy one machine and disassemble it to learn how it was built and how it works. A chemical company may use reverse engineering to defeat a patent on a competitor's manufacturing process. In civil engineering, bridge and building designs are copied from past successes so there will be less chance of catastrophic failure. In software engineering, good source code is often a variation of other good source code.

Reverse engineering is a specific approach that allows to effectively link the measurement technique, laser or CT scanners, with a computer. This type of connection allows to transform the scanned data into the digital form and process them with the computer. Reverse engineering is known as the process of duplicating an existing component sub-assembly or product without using a drawing documentation or a computer model $[1,2]$.

The laser scanning has a many application in the field of the engineering industry. A laser beam is projected onto a surface and the reflected beam is detected by CCD

\footnotetext{
* Corresponding author: Ondrej.Stalmach@fstroj.uniza.sk

Reviewers: Wiesława Piekarska, Krzysztof Talaśka
} 
cameras. Using the image processing and triangulation method are acquired threedimensional coordinates of the cloud of points [3].

The external surface of an object is represented with a set of discrete points. Individual points are described in the file with their $3 \mathrm{D}$ coordinates. The density of the points on the surface is optional at certain intervals, for example in the engineering industry it is roughly 1 to $0.2 \mathrm{~mm}$ between two points.

Triangulation is a method which uses location and angles between light sources and photo sensing devices to deduce position. A high energy light source is focused and projected at a prespecified angle at the surface of interest [4]. It forms the basis for algorithms that are applied to create the cloud of points. After scanning the object with a laser scanner, it is necessary to process the obtained cloud of points. It is done by trigonometric calculation. The solution is to connect individual points together to a mesh that describes the geometry of the scanned object. Nowadays, there are several meshing algorithms that enable us to solve this type of problem.

\section{Poisson Surface Reconstruction}

Michael Kazhdan in his paper said: We show that surface reconstruction from oriented points can be cast as a spatial Poisson problem. This Poisson formulation considers all the points at once, without resorting to heuristic spatial partitioning or blending, and is therefore highly resilient to data noise. Unlike radial basis function schemes, our Poisson approach allows a hierarchy of locally supported basis functions, and therefore the solution reduces to a well-conditioned sparse linear system. We describe a spatially adaptive multiscale algorithm whose time and space complexities are proportional to the size of the reconstructed model [5].

This contribution is based on this algorithm too because the aim is to get a watertight and smooth surface of the model. Therefore the software MeshLab is use because it has implemented the Poisson Surface Reconstruction mesh algorithm.

\section{Processing of scanned data and modelling}

After scanning an object into the cloud of points is created a mesh from these points. As the reference object for this study is used the wrench from the chrome-vanadium steel, size 30. It is scanned using the laser FARO Edge ScanArm HD in the company CEIT. At the output from the scanner is received a text file that contained the coordinates of cloud of points, defined in the cartesian coordinate system XYZ.

In the Figure 1 is denoted the whole workflow. After scanning the wrench using the laser scanner is the text file containing the positions of the points imported to the freeware software MeshLab. In this software is created a triangular surface model in the format .stl. In another freeware software MeshMixer are removed the information like size of the wrench and material from the surface of the model by the smoothing tool. After that the two different approaches of further processing of the model are studied.

The first approach is the classic reverse engineering approach which includes the creation of the volume CAD model, because the .stl model is only the triangular surface model.

Volume CAD model is created in the software Ansys SpaceClaim. After importing the .stl model into the SpaceClaim, the main boundary curves of the future volume model are created with the using of intersection of $2 \mathrm{D}$ planes and triangular surface model. Using these boundary curves the volume model is created by re-modelling the triangular surface 
model. The volume model must be created as close as possible to the triangulated surface model. Then the volume model is used in the FEM analysis [6-9].

The second approach doesn't include the creation of volume CAD model. The stl model is imported to the module ICEM CFD which is implemented in the Ansys Workbench and it is effective tool for generating a FEM meshes. This tool is used to create the surface mesh from the triangular surface model. After that this surface mesh is used to compute the volume mesh. Then the volume mesh is used in the FEM analysis.

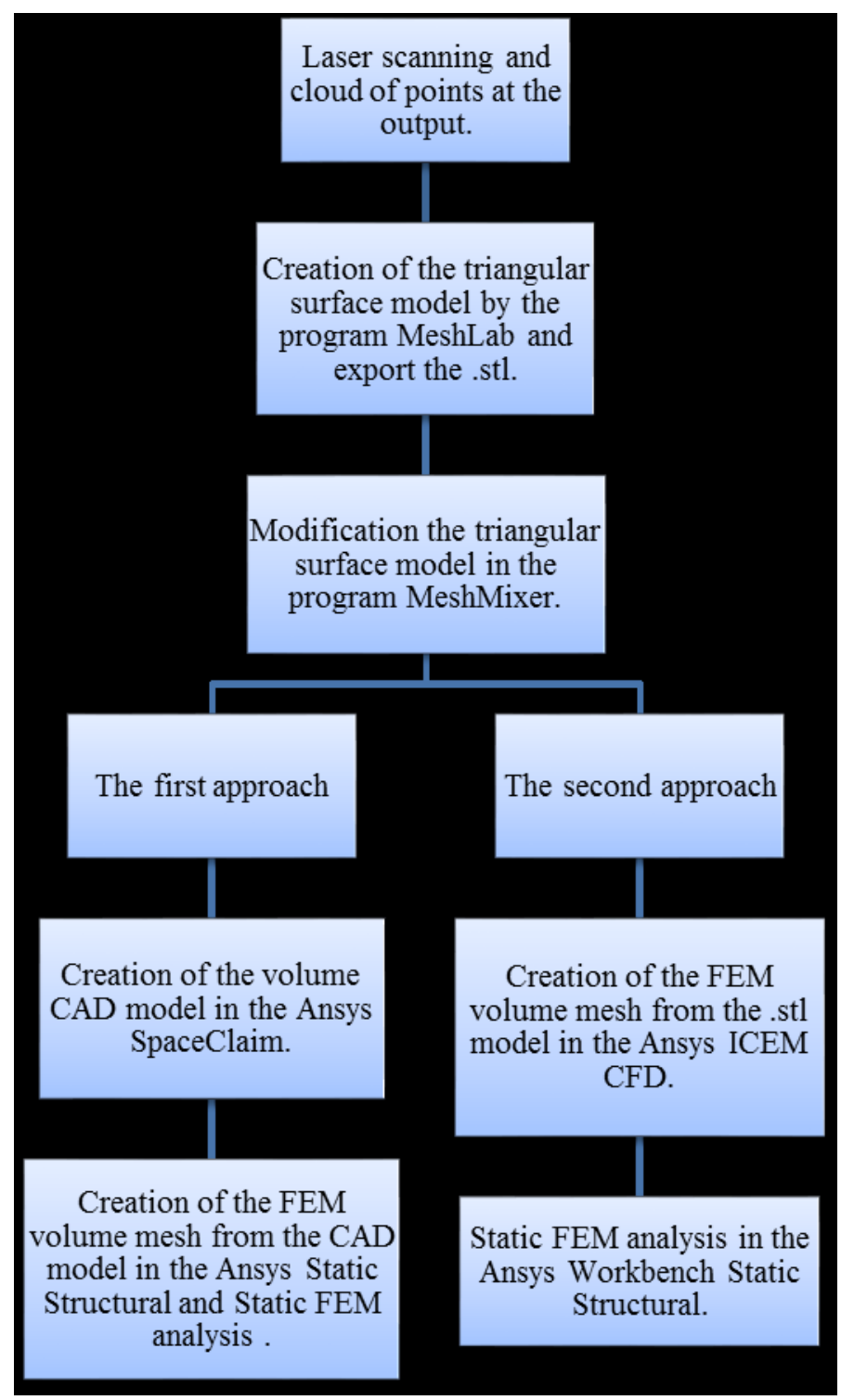

Fig. 1. Scheme of the workflow 


\section{FEM Analysis}

The FEM models from these two approaches are simulated in the software Ansys Workbench in the module Static Structural. The purpose of this paper is to compare the simulations of these two approaches therefor the simple and the same boundary conditions are set on the these both FEM models.

In the Figure 2 is shown that the two parallel surfaced of the wrench are fixed and on the other side of the wrench is applied the pressure $0.59 \mathrm{MPa}$ in the direction of the movement.

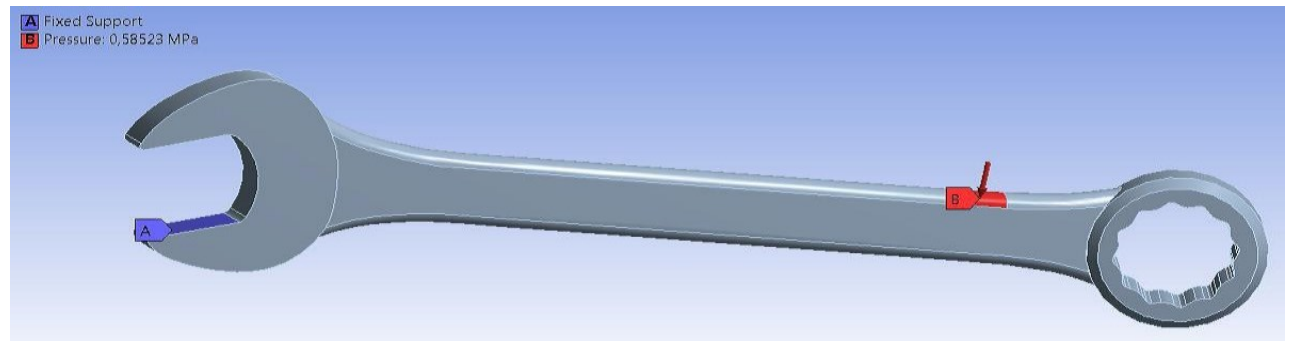

Fig. 2. Location of boundary conditions

Both simulations are completed successfully and the results are shown on the Figure 3 and 4.

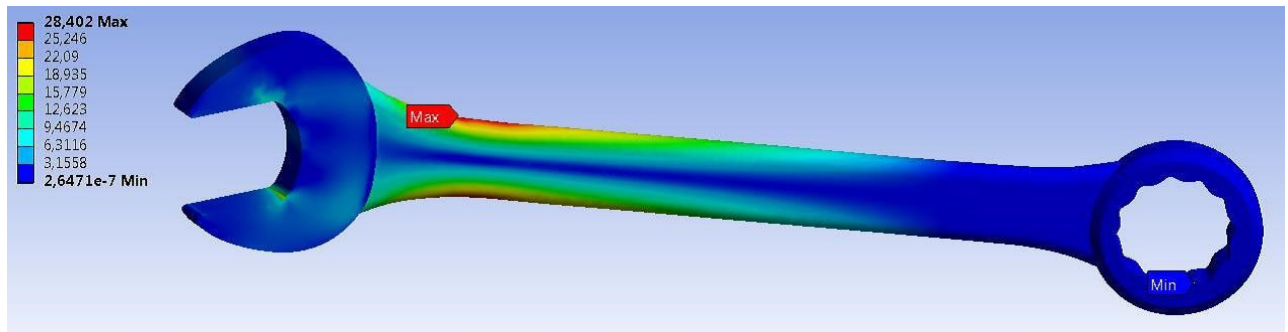

Fig. 3. Result of 1. Approach - Equivalent von-Mises stress

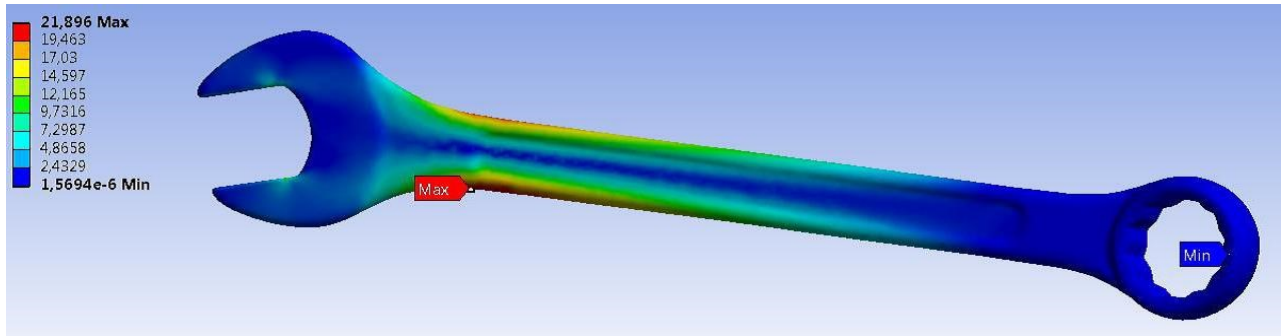

Fig. 4. Result of 2. Approach - Equivalent von Mises stress

The results are different. Maximum value of the $1^{\text {st }}$ approach von Mises stress is place at the different locations than the maximum value of the $2^{\text {nd }}$ approach von Mises stress and the deviation of the results is $29,7 \%$. The reason why the results are different may be the fact that the geometry of volume CAD model which is the first approach is a little different like reference wrench because it is created by re-modelling from the triangulated surface model. Therefor it can't be the $100 \%$ same as the original triangular surface model. A complete comparison of the approaches would require comparison with an experiment at which deformations would be measured on a loaded object. One of the suitable methods is, for 
example, optical determination of deformation by image recognition or random raster, respectively [10-12].

\section{Conclusions}

The two different approaches are compared while the first one included the creation of the volume CAD model and the other one not. The results of FEM analysis are different but the 2nd approach may have better results because that geometry is almost exact copy of the real reference wrench. The 1st approach have a little different geometry in compare to the original. At this problem is looked from the view of FEM analyze and not from the view of classic reverse engineering. Classic reverse engineering need to create the volume CAD model because it is used for another applications not only for the FEM analyze. From the view of the FEM analyze is the 2nd approach better because do not need to create the volume CAD model. When the volume CAD model is needed the 2 nd approach is useless. But in the situations when only the FEM analyze is needed the 2nd approach is faster than the first one.

The part of the results of this work has been supported by VEGA grant No. 1/0795/16 and KEGA grant No. 017ŽU-4/2017.

\section{References}

1. O. Stalmach, Implementation and verification of data conversion from the laser scanner to the FEM. Diploma thesis, Zilina: University of Zilina, 59 (2017)

2. J. Bocko, P. Lengvarsky, Application of Finite Element Method for Analysis of Nanostructures. Acta mechanica et automatica, 11, 116-120 (2017)

3. S. Son, H. Park, J. Lee, Automated laser scanning system for reverse engineering and inspection. International Journal of Machine Tools and Manufacture, 42, 889-897 (2002)

4. T. Varady, RR. Martin, J. Cox, Reverse engineering of geometric models and introduction. Computer Aided Design, 29, 255-268 (1997)

5. M. Bolitho, M. Kazhdan, R. Burns, H. Hoppe, Parallel Poisson Surface Reconstruction. Advances in Visual Computing, 678-685 (2009)

6. B. Leitner, M. Vaško, Design and Modelling of Tank Wagon Assembly Operations in CAM Environment. Transport Means 2015 I \& II, 87-90 (2015)

7. M. Macko, K. Tyszczuk, G. Śmigielski, J. Flizikowski, A. Mroziński, The use of CAD applications in the design of shredders for polymers. MATEC, 157 (2018)

8. M. Macko, J. Flizikowski, Z. Szczepański, K. Tyszczuk, G. Śmigielski, A. Mroziński, J. Czerniak, A. Tomporowski, CAD/CAE applications in mill's design and investigation. Lecture Notes in Mechanical Engineering, 343-351 (2017)

9. L. Jakubovičová, M. Sága, M. Vaško, Numerical study of influence of mutual slewig of roller bearing rings on the principal stresses at contact area. Scientific journal of Silesian University of Technology - Series Transport 84, 83-91 (2014)

10. M. Kubiak, T. Domanski, V. Dekys, A. Sapietova, Measurement of strain during tension test of welded joint using multi-camera $3 D$ correlation system. Procedia Engineering, 177, 107-113 (2017)

11. P. Krawiec, G. Domek, Ł. Warguła, K. Waluś, J. Adamiec, The application of the optical system ATOS II for rapid prototyping methods of non-classical models of cogbelt pulleys. MATEC Web of Conferences, 157, 01010 (2018) 
12. J. Sobotka, P. Solfronk, M. Kolnerova, L. Zuzanek, Utilization of the high-speed cameras for monitoring deformation behaviour at the bending impact test. Proc. METAL, 482-487 (2016) 